\title{
NUEVO CRITERIO CONTABLE BASADO EN LA INFORMACIÓN PROSPECTIVA
}

\author{
NEW ACCOUNTING STANDARD INFORMATION BASED PROSPECTIVE
}

\section{Elsa Esther Choy Zevallos*}

Docente Asociado de la Facultad de Ciencias Contables- UNMSM

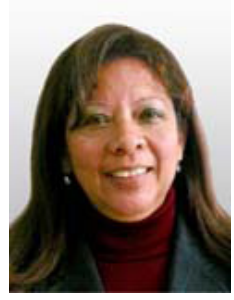

[Recepción: Setiembre de 2011/ Conformidad: Noviembre de 2011]

\section{RESUMEN}

Hoy las decisiones se toman sobre la base de información del presente y del futuro; no es suficiente la información contenida en los estados contables históricos, elaborada y publicada con relación a lo ocurrido en el pasado. Para los agentes económicos la información particularmente importante es la que les permite analizar y considerar las posibilidades de eventos futuros que deberá afrontar la empresa.

Existen evidencias relacionadas con la información contable prospectiva, pero aplicándose parcialmente, en forma asistemática y sin adoptar un modelo determinado.

El presente artículo, presenta la siguiente hipótesis "Los estados de información prostectiva deben estar preparados sobre la base de criterios consistentes con los periodos anteriores, aplicando las normas contables que permitan verificar con cierto grado de razonabilidad las situaciones contingentes que deberán afrontar las empresas”.

El objetivo es establecer la necesidad de elaborar información contable prospectiva, incorporando principios contables para las estimaciones, que permita mostrar eventos futuros; es así que se determinaria que mediante la información contable prospectiva se logra vincular la información del pasado y las perspectivas futuras de la empresa.

El diseño de la investigación es de carácter descriptivo ya que pretende señalar, analizar, establecer y evaluar los procesos de proyección para la información contable prospectiva.

Palabras Clave: Información contable, Prospectiva organizacional, Principios Contables, Información Prospectiva

\begin{abstract}
Todaydecisions are madeon the basisof informationpresent andfutureis not enoughinformation inthe historical financial statements, prepared and publishedin relationto what happened inthe past.For economic agentsisparticularly important informationthat enables them toanalyze and considerthe possibilitiesof future events thatwill facethe company. Evidencerelating toprospectivefinancial information, but partiallyappliedinpiecemealandwithout adoptinga particular model.

Thispaper presents thefollowing hypothesis"states prostectivainformationshould be preparedon the basis ofcriteria consistent withprior periods, the accounting rulesapplyingto verifywith any degree ofreasonablenessthecontingenciesthatwill facebusinesses." The aimis to establishthe need forprospectivefinancial information, incorporating accountingfor estimates, for displayingfuture events, sothatwould determinewhichprospectivefinancial informationbylinking informationis achievedin the past andthe future prospects ofthe company.

Theresearch designis descriptiveas itseeks to identify, analyze, and evaluate the processesofscreening for theprospectivefinancial information.
\end{abstract}

Keywords: Accounting, Prospective organizational, Accounting Principles, Prospective information

* Doctora en Ciencias Contables y Empresariales - UAP. Magister en Dirección Financiera - USMP. Contadora Publica Colegiada Certificada. Docente Investigadora de la Facultad de Ciencias Contables - UNMSM.

E-mail: eschoyz@yahoo.com 


\section{INTRODUCCIÓN}

La información que utilizan los agentes económicos, entre las que ocupa un lugar destacado la contabilidad, ha experimentado un considerable avance despues de la Segunda Guerra Mundial, principalmente con la informática. Mientras que desde un primer momento la planificación financiera se fue enriqueciendo gradualmente con el aporte de estos valiosos instrumentos de planificación, la contabilidad parecía relegada a un papel secundario. Pero la realidad puso en evidencia que la contabilidad constituye todavía el mejor sistema de información empresarial ideado por el hombre.

Estamos convencidos de poder afirmar que cualquier modelo de planificación empresarial que se elabore al margen de la contabilidad es, cuando menos, incompleto.

En este contexto, corresponde hacer referencia al origen y evolución de los modelos matemáticos aplicados para planificación en la empresa, entre los modelos teóricos que han sido presentados en su oportunidad a la comunicad científica internacional, en primer término y que luego han ejercido una notable influencia en el relanzamiento de nuevas líneas de investigación en el área de las ciencias contables y de la administración, cabe mencionar a los siguientes:

Charnes, Cooper y Miller (1959) Planificación de almacenes con restricciones financieras.

Richard Mattersich (1961), de gestión presupuestaria, que influyó en muchos de los modelos de planificación que se elaboraron posteriormente.

D.I Chambers (1967) Planificación de inversiones con restricciones presupuestarias y cumplimiento de ratios

W.T.Carleton (1970) Macro modelo para la actividad financiera de la empresa y modelo de planificación financiera a largo plazo.

\section{INFORMACIÓN PROSPECTIVA}

La Norma Internacional sobre Compromiso de Aseguramiento (NICA) 3400, señala:

"Información financiera prospectiva" significa información financiera basada en estimaciones sobre eventos que pueden ocurrir en el futuro y posibles acciones de una organización. Es altamente subjetiva en naturaleza y su preparación requiere el ejercicio de considerable criterio. La información financiera prospectiva puede tomar la forma de pronóstico, una proyección o una combinación de ambos, por ejemplo, un pronóstico de un año más una proyección de cinco años"

La utilidad de la información prospectiva se justifica por su valor para ayudar a la toma de decisiones económicas. Disponer de estimaciones razonables sobre la evolución futura de la empresa, a pesar de las incertidumbres que inevitablemente deben afrontarse, tiene una importancia innegable para los usuarios.

La información prospectiva también adquiere gran relevancia para calibrar las posibilidades que tiene el negocio de seguir en marcha y cumplir con el principio de gestión continuada.

Muchas empresas, con la finalidad de informar o crear expectativas a los distintos usuarios, publican previsiones sobre resultados y otras magnitudes financieras que estiman alcanzar en un horizonte temporal más o menos amplio. Esta práctica no está restringida a las empresas cuyos títulos cotizan en el mercado, pero es particularmente importante en las empresas cotizadas, porque las proyecciones publicadas pueden llegar a tener efectos significativos en los precios de los títulos.

En relación con la información contable o financiera de carácter prospectivo, y para evitar conflictos de intereses, deben respetarse, al menos algunos principios. 


\section{PRINCIPIOS DE CARÁCTER GENERAL}

a. La información prospectiva es relevante para el mercado y, por tanto, debe ser veraz, clara, lo más completa posible y, cuando lo exija la naturaleza de los datos, cuantificada e incluir advertencias sobre su grado de certidumbre, para no inducir a confusión o engaño a los usuarios.

b. La información prospectiva tiene que ser de carácter voluntario, ya que ha de basarse en los planes asumidos y las proyecciones realizadas internamente por la empresa. Sin embargo, las previsiones que la empresa decida publicar deben elaborarse de forma coherente con las políticas contables aplicadas en la formulación de las cuentas anuales y ser contrastables con la información real, para facilitar a los usuarios su seguimiento $y$ evaluación.

c. La presentación de información prospectiva debe diferenciarse claramente si se trata de objetivos que maneja la empresa o estimaciones sobre la evolución esperada de sus negocios. Igualmente, debe identificarse el horizonte temporal que abarcan las proyecciones, así como la naturaleza y razonabilidad de las asunciones e hipótesis en las que se sustenta.

d. Respecto a la actualización y rectificación de previsiones, cuando la información prospectiva es buena, el mercado dispone de los datos necesarios para evaluar la calidad de las estimaciones y está en situación de interpretar la incidencia de cualquier acontecimiento externo. Si se producen desviaciones significativas en las hipótesis de partida o en las proyecciones financieras, la empresa debe actualizar o rectificar las previsiones inmediatamente, con el fin de transmitir al mercado las señales oportunas. Una manera de juzgar la bondad de la información prospectiva publicada será presentar cada año las diferencias registradas durante el ejercicio anterior entre las previsiones y los datos reales. Esta práctica ayuda a los usuarios a analizar la calidad de los pronósticos empresariales y a precisar el nivel de confianza que puede depositar en dichas estimaciones.

e. Algunas veces la proyección de estados financieros completos puede ser considerada por las empresas como información reservada. Sin embargo, las empresas pueden estar interesadas en publicar previsiones condensadas o centradas en magnitudes concretas que son relevantes para la evolución de sus negocios, es lo que se conoce como información prospectiva de variables.

La ausencia de una normativa específica que regule los procedimientos de divulgación y el contenido de la información prospectiva, dificulta su seguimiento por parte de los organismos supervisores, no permite la comparación con los datos publicados por otras empresas del sector, incluso con los estados financieros históricos de la propia empresa, y su difusión puede ocasionar eventuales conflictos de intereses por sus efectos en las decisiones económicas de los inversores y otros usuarios.

\section{OBJETIVOS DE LOS ESTADOS CONTABLES PROYECTADOS}

Los objetivos que debe cumplir la información contable prospectiva son:

a. Lograr ajustar los resultados del ejercicio, ya que se puede recoger información posterior al cierre.

b. Aplicar racionalmente la asignación de ganancias acumuladas de manera que cubran las necesidades financieras futuras del negocio.

c. Demostrar que la empresa se encuentra en marcha y tiene perspectivas futuras.

d. Contribuir a orientar la selección de alternativas para el negocio, facilitando la inclusión de la perspectiva Responsabilidad Social Empresarial como aspecto integrador significativo. 
e. Contribuir a reducir los riesgos de toma de decisiones e interpretar información financiera de los negocios, por parte de los usuarios ajenos del negocio.

Para poder cumplir con estos objetivos, la información proyectada deberá diseñarse tomando como guía algunos requisitos.

\section{REQUISITOS DE LA INFORMACIÓN PROSPECTIVA}

a. Conceptos básicos:

- Empresa en Marcha

- Utilidad

b. Requisitos generales:

- Oportunidad

- Viabilidad

- Totalidad

- Comparabilidad

- Uniformidad

- Confidencialidad

- Exposición

- Devengado

c. Requisitos específicos:

- Prudencia

\section{MODELO CONTABLE APLICABLE A LA INFORMACIÓN PROSPECTIVA}

El diseño de la información contable prospectiva debe guardar relación con el modelo utilizado para presentar la situación económico-financiera de cierre de ejercicio.

El modelo debe incluir definiciones sobre:

- Capital a mantener

- Unidad de medida

- Criterios de mediciones

- Tasa de descuentos de flujos futuros de fondos esperados.

El modelo construido sobre la base de:

- Capital a mantener: Capital Financiero

- Unidad de medida: La moneda, ajustada en su caso, en su poder adquisitivo.

- Criterios de mediciones: Criterios de proyección de costos, de absorción total.
- Tasa de descuento: Resulta adecuado usar la tasa a la que accede la empresa, en lugar de la tasa de mercado cuya estimación es extremadamente subjetiva.

Es importante reiterar que el modelo contable debe ser consistente para la información contable histórica y la información contable prospectiva.

\section{CONCLUSIONES Y RECOMENDACIONES}

1. La información contable proyectada o prospectiva es indispensable para mejorar la información histórica que presenta los estados financieros destinados a terceros, y facilitar las comparaciones entre ambas informaciones.

2. Se plantea la necesidad de formular información contable de carácter prospectiva tomando como base criterios consistentes con los periodos anteriores, aplicando normas contables que permitan verificar con cierto grado de razonabilidad las situaciones contingentes que deberán afrontar las empresas.

\section{REFERENCIAS BIBLIOGRÁFICAS}

1. BIONDI, M (2007) "los efectos de los cambios de escenarios sobre las bases teóricas para la preparación de la información contable proyectada o prospectiva”. Universidad Buenos Aires. Argentina

2. VIEGAS, J. (2000) "La información Prospectiva: Proyecciones o Pronósticos". Contabilidad y Auditoría. Número 12-Año 6. Instituto de Investigaciones Contables. Facultad de Ciencias Económicas. UBA. 2000.

3. Primer y Segundo Informe de Avance Proyecto UBACyT E037 "Los efectos de los cambios de escenario sobre las bases teóricas para la preparación de la información contable proyectada o prospectiva” Años 2004 y 2005.

4. "Escenario Microeconómico y Macroeconómico Argentino” por Juan Carlos de Pablo en www.depabloconsult.com.ar (visitado $12 / 10 / 11$ ) 